\title{
Rapidity Distributions in Drell-Yan and Higgs Productions at Threshold to Third Order in QCD
}

\author{
Taushif Ahmed, ${ }^{1, *}$ M. K. Mandal, ${ }^{1, \dagger}$ Narayan Rana, ${ }^{1, \ddagger}$ and V. Ravindran ${ }^{2, \S}$ \\ ${ }^{1}$ Regional Centre for Accelerator-based Particle Physics, Harish-Chandra Research Institute, Allahabad, India \\ ${ }^{2}$ The Institute of Mathematical Sciences, Chennai, India \\ (Received 6 May 2014; published 20 November 2014)
}

\begin{abstract}
We present the threshold $\mathrm{N}^{3} \mathrm{LO}$ perturbative QCD corrections to the rapidity distributions of dileptons in the Drell-Yan process and Higgs boson in gluon fusion. Sudakov resummation of QCD amplitudes, renormalization group invariance, and the mass factorization theorem provide useful guidelines to obtain them in an elegant manner. We use various state of the art three loop results that have been recently available to obtain these distributions. For the Higgs boson, we demonstrate numerically the importance of these corrections at the LHC.
\end{abstract}

DOI: 10.1103/PhysRevLett.113.212003

PACS numbers: $12.38 . \mathrm{Bx}$

Drell-Yan (DY) production [1] of a pair of leptons at the Large Hadron Collider (LHC) is one of the cleanest processes that can be studied not only to test the standard model (SM) to an unprecedented accuracy but also to probe physics beyond the SM (BSM) scenarios in a very clear environment. Rapidity distributions of $Z$ boson [2] and charge asymmetries of leptons in $W$ boson decays [3] constrain various parton densities and, in addition, possible excess events can provide hints to BSM physics, namely, $R$ parity violating supersymmetric models, models with $Z^{\prime}$ or with contact interactions and large extra-dimension models. One of the production mechanisms responsible for discovering the Higgs boson of the SM at the LHC [4] is the gluon-gluon fusion through top quark loop. Being a dominant one, it will continue to play a major role in studying the properties of the Higgs boson and its coupling to other SM particles. Distributions of transverse momentum and rapidity of the Higgs boson are going to be very useful tools to achieve this task. Like the inclusive rates $[5,6]$, the rapidity distribution of dileptons in DY process and of the Higgs boson in gluon-gluon fusion are also known to next to next to leading order (NNLO) level in perturbative QCD due to seminal works by Anastasiou et al. [7]. The quark and gluon form factors [8-10], the mass factorization kernels [11], and the renormalization constant [12] for the effective operator describing the coupling of the Higgs boson with the SM fields in the infinite top quark mass limit up to three loop level in dimensional regularization with space-time dimensions $n=4+\epsilon$ were found to be useful to obtain the next to next to next to leading order $\left(\mathrm{N}^{3} \mathrm{LO}\right)$ threshold effects [13] to the inclusive Higgs boson and DY productions at the LHC, excluding $\delta(1-z)$ terms, where the scaling parameter is $z=m_{l^{+} l^{-}}^{2} / \hat{s}$ for the DY process and $z=m_{H}^{2} / \hat{s}$ for the Higgs boson. Here, $m_{l^{+} l^{-}}, m_{H}$ and $\hat{s}$ are the invariant mass of the dileptons, the mass of the Higgs boson, and center of mass energy of the partonic reaction responsible for the production mechanism, respectively. Recently, Anastasiou et al. [14] made an important contribution in computing the total rate for the Higgs boson production at $\mathrm{N}^{3} \mathrm{LO}$ resulting from the threshold region including the $\delta(1-z)$ term. Their result, along with three loop quark form factors and mass factorization kernels, was used to compute the DY cross section at $\mathrm{N}^{3} \mathrm{LO}$ at threshold in [15].

In this Letter, we will apply the formalism developed in [16] to obtain rapidity distributions of the dilepton pair and of the Higgs boson at $\mathrm{N}^{3} \mathrm{LO}$ in the threshold region using the available information that led to the computation of the $\mathrm{N}^{3} \mathrm{LO}$ threshold corrections to the inclusive Higgs boson and DY productions.

The rapidity distribution can be written as

$$
\frac{d \sigma^{I}}{d y}=\sigma_{\mathrm{Born}}^{I}\left(x_{1}^{0}, x_{2}^{0}, q^{2}\right) W^{I}\left(x_{1}^{0}, x_{2}^{0}, q^{2}\right), \quad I=q, g,
$$

normalized by $W_{\mathrm{Born}}^{I}\left(x_{1}^{0}, x_{2}^{0}, q^{2}\right)=\delta\left(1-x_{1}^{0}\right) \delta\left(1-x_{2}^{0}\right)$. Rapidity $\quad y=\frac{1}{2} \log \left(p_{2} \cdot q / p_{1} \cdot q\right)=\frac{1}{2} \log \left(x_{1}^{0} / x_{2}^{0}\right) \quad$ and $\tau=q^{2} / S=x_{1}^{0} x_{2}^{0}, q$ being the momentum of the dilepton pair in the DY process and of the Higgs boson in the Higgs boson production, $S=\left(p_{1}+p_{2}\right)^{2}$, where $p_{i}$ are the momenta of incoming hadrons $P_{i}(i=1,2)$. For the DY process, $I=q$ and $\sigma^{I}=d \sigma^{q}\left(\tau, q^{2}, y\right) / d q^{2}$ with $q^{2}$ the invariant mass of the final state dilepton pair, i.e., $q^{2}=$ $m_{l^{+} l^{-}}^{2}$ and for the Higgs boson production through gluon fusion, $I=g$ and $\sigma^{I}=\sigma^{g}\left(\tau, q^{2}, y\right)$. The function $W^{I}$ can be expressed in terms of the parton distribution functions $f_{a}\left(x_{1}, \mu_{F}^{2}\right)$ and $f_{b}\left(x_{2}, \mu_{F}^{2}\right)$ renormalized at the factorization scale $\mu_{F}$,

$$
\begin{aligned}
W^{I}= & \sum_{a b=q, \bar{q}, g} \int_{x_{1}^{0}}^{1} \frac{d z_{1}}{z_{1}} \int_{x_{2}^{0}}^{1} \frac{d z_{2}}{z_{2}} \mathcal{H}_{a b}^{I}\left(\frac{x_{1}^{0}}{z_{1}}, \frac{x_{2}^{0}}{z_{2}}, \mu_{F}^{2}\right) \\
& \times \Delta_{d, a b}^{I}\left(z_{1}, z_{2}, q^{2}, \mu_{F}^{2}, \mu_{R}^{2}\right),
\end{aligned}
$$


with

$$
\begin{aligned}
& \mathcal{H}_{a b}^{q}\left(x_{1}, x_{2}, \mu_{F}^{2}\right)=f_{a}^{P_{1}}\left(x_{1}, \mu_{F}^{2}\right) f_{b}^{P_{2}}\left(x_{2}, \mu_{F}^{2}\right), \\
& \mathcal{H}_{a b}^{g}\left(x_{1}, x_{2}, \mu_{F}^{2}\right)=x_{1} f_{a}^{P_{1}}\left(x_{1}, \mu_{F}^{2}\right) x_{2} f_{b}^{P_{2}}\left(x_{2}, \mu_{F}^{2}\right),
\end{aligned}
$$

where $x_{i}(i=1,2)$ are the momentum fractions of the partons in the incoming hadrons. The threshold contribution to the rapidity distribution denoted by $\Delta_{d, I}^{\mathrm{SV}}\left(z_{1}, z_{2}, q^{2}, \mu_{R}^{2}, \mu_{F}^{2}\right)$ is found to be

$$
\Delta_{d, I}^{\mathrm{SV}}=\left.\mathcal{C} \exp \left(\Psi_{d}^{I}\left(q^{2}, \mu_{R}^{2}, \mu_{F}^{2}, \bar{z}_{1}, \bar{z}_{2}, \epsilon\right)\right)\right|_{\epsilon=0},
$$

where $\Psi_{d}^{I}$ are finite distributions computed in $4+\epsilon$ spacetime dimensions with $\bar{z}_{1}=1-z_{1}$ and $\bar{z}_{2}=1-z_{2}$ :

$$
\begin{aligned}
\Psi_{d}^{I}= & \left(\ln \left(Z^{I}\left(\hat{a}_{s}, \mu_{R}^{2}, \mu^{2}, \epsilon\right)\right)^{2}\right. \\
& \left.+\ln \left|\hat{F}^{I}\left(\hat{a}_{s}, Q^{2}, \mu^{2}, \epsilon\right)\right|^{2}\right) \delta\left(\bar{z}_{1}\right) \delta\left(\bar{z}_{2}\right) \\
& +2 \Phi_{d}^{I}\left(\hat{a}_{s}, q^{2}, \mu^{2}, \bar{z}_{1}, \bar{z}_{2}, \epsilon\right) \\
& -\mathcal{C}\left(\ln \Gamma_{I I}\left(\hat{a}_{s}, \mu^{2}, \mu_{F}^{2}, \bar{z}_{1}, \epsilon\right) \delta\left(\bar{z}_{2}\right)+\left(\bar{z}_{1} \leftrightarrow \bar{z}_{2}\right)\right) .
\end{aligned}
$$

The definition of double Mellin convolution $\mathcal{C}$ can be found in [16]. We drop all the regular functions that result from various convolutions. The bare form factors are denoted by $\hat{F}^{I}$ with $Q^{2}=-q^{2}$. The overall operator renormalization constant for the DY process, $Z^{q}=1$ and for the Higgs boson, $Z^{g}$ is known up to the three loop level [12] in QCD. $\Phi_{d}^{I}$ are called the soft distribution functions and $\Gamma_{I I}$ are the mass factorization kernels. $\mu$ is the scale introduced to define the dimensionless strong coupling constant $\hat{a}_{s}=$ $\hat{g}_{s}^{2} / 16 \pi^{2}$ in dimensional regularization and $a_{s}\left(\mu_{R}^{2}\right)$ is the renormalized strong coupling constant which is related to $\hat{a}_{s}$ through the renormalization constant $Z\left[a_{s}\left(\mu_{R}^{2}\right)\right]$, i.e., $\hat{a}_{s}=\left(\mu / \mu_{R}\right)^{\epsilon} Z\left(\mu_{R}^{2}\right) S_{\epsilon}^{-1} a_{s}\left(\mu_{R}^{2}\right), S_{\epsilon}=\exp \left[\left(\gamma_{E}-\ln 4 \pi\right) \epsilon / 2\right]$. The fact that $\Delta_{d, I}^{\mathrm{SV}}$ are finite in the limit $\epsilon \rightarrow 0$ implies that the pole structure of the soft distribution functions should be similar to that of $\hat{F}^{I}$ and $\Gamma_{I I}$. We find that they must satisfy Sudakov type differential equations which the form factors $\hat{F}^{I}$ also satisfy:

$$
q^{2} \frac{d}{d q^{2}} \Phi_{d}^{I}=\frac{1}{2}\left[\bar{K}_{d}^{I}+\bar{G}_{d}^{I}\right]
$$

where the constants $\bar{K}_{d}^{I}\left(\hat{a}_{s},\left(\mu_{R}^{2} / \mu^{2}\right), \bar{z}_{1}, \bar{z}_{2}, \epsilon\right)$ are proportional to the singular terms in $\epsilon$ and the $\bar{G}_{d}^{I}\left(\hat{a}_{s},\left(q^{2} / \mu_{R}^{2}\right)\right.$, $\left.\left(\mu_{R}^{2} / \mu^{2}\right), \bar{z}_{1}, \bar{z}_{2}, \epsilon\right)$ are finite functions of $\epsilon$. It is straightforward to solve the above differential equations yielding

$$
\Phi_{d}^{I}=\sum_{i=1}^{\infty} \hat{a}_{s}^{i}\left(\frac{q^{2} \bar{z}_{1} \bar{z}_{2}}{\mu^{2}}\right)^{i(\epsilon / 2)} S_{\epsilon}^{i}\left(\frac{(i \epsilon)^{2}}{4 \bar{z}_{1} \bar{z}_{2}}\right) \hat{\phi}_{d}^{I,(i)}(\epsilon),
$$

where

$$
\hat{\phi}_{d}^{I,(i)}(\epsilon)=\frac{1}{i \epsilon}\left[\bar{K}_{d}^{I,(i)}(\epsilon)+\bar{G}_{d}^{I,(i)}(\epsilon)\right]
$$

The constants $\bar{K}_{d}^{I,(i)}(\epsilon)$ are determined by expanding $\bar{K}_{d}^{I}$ in powers of the bare coupling constant $\hat{a}_{s}$, i.e.,

$$
\bar{K}_{d}^{I}=\delta\left(\bar{z}_{1}\right) \delta\left(\bar{z}_{2}\right) \sum_{i=1}^{\infty} \hat{a}_{s}^{i}\left(\frac{\mu_{R}^{2}}{\mu^{2}}\right)^{i(\epsilon / 2)} S_{\epsilon}^{i} \bar{K}_{d}^{I,(i)}(\epsilon),
$$

and solving the RG equation for $\bar{K}_{d}^{I}$. We find that $\bar{K}_{d}^{I,(i)}(\epsilon)$ are identical to $\bar{K}^{I,(i)}(\epsilon)$ given in [17]. The constants $\bar{G}_{d}^{I,(i)}(\epsilon)$ are related to the finite boundary functions $\bar{G}_{d}^{I}\left(\hat{a}_{s}, 1,\left(q^{2} / \mu^{2}\right), \bar{z}_{1}, \bar{z}_{2}, \epsilon\right)$. Defining the $\overline{\mathcal{G}}_{d, i}^{I}(\epsilon)$ through the relation

$$
\sum_{i=1}^{\infty} \hat{a}_{s}^{i}\left(\frac{q^{2} \bar{z}_{1} \bar{z}_{2}}{\mu^{2}}\right)^{i(\epsilon / 2)} S_{\epsilon}^{i} \bar{G}_{d}^{I,(i)}(\epsilon)=\sum_{i=1}^{\infty} a_{s}^{i}\left(q^{2} \bar{z}_{1} \bar{z}_{2}\right) \overline{\mathcal{G}}_{d, i}^{I}(\epsilon)
$$

and demanding the finiteness of $\Delta_{d, I}^{\mathrm{SV}}$ given in Eq. (4), we find that the structure of $\overline{\mathcal{G}}_{d, i}^{I}(\epsilon)$ is similar to that of the corresponding $G^{I}(\epsilon)$ in the form factors [17], that is

$$
\overline{\mathcal{G}}_{d, i}^{I}(\epsilon)=-f_{i}^{I}+\bar{C}_{i}^{I}+\sum_{k=1}^{\infty} \epsilon^{k} \overline{\mathcal{G}}_{d, i}^{I, k},
$$

where $\quad \bar{C}_{1}^{I}=0, \quad \bar{C}_{2}^{I}=-2 \beta_{0} \overline{\mathcal{G}}_{d, 1}^{I, 1}, \quad \bar{C}_{3}^{I}=-2 \beta_{1} \overline{\mathcal{G}}_{d, 1}^{I, 1}-$ $2 \beta_{0}\left(\overline{\mathcal{G}}_{d, 2}^{I, 1}+2 \beta_{0} \overline{\mathcal{G}}_{d, 1}^{I, 2}\right), f_{i}^{I}$ are given in [8] and $\beta_{i}$ are the coefficients of the QCD $\beta$ function of $a_{s}\left(\mu_{R}^{2}\right), \mu_{R}^{2} d a_{s}\left(\mu_{R}^{2}\right) /$ $d \mu_{R}^{2}=\epsilon a_{s}\left(\mu_{R}^{2}\right) / 2-\sum_{i=0}^{\infty} \beta_{i} a_{s}^{i+2}\left(\mu_{R}^{2}\right)$. The constants $\overline{\mathcal{G}}_{d, i}^{I, k}$ can be expressed in terms of $\overline{\mathcal{G}}_{i}^{I, k}$ using the following relation

$$
\int_{0}^{1} d x_{1}^{0} \int_{0}^{1} d x_{2}^{0}\left(x_{1}^{0} x_{2}^{0}\right)^{N-1} \frac{d \sigma^{I}}{d Y}=\int_{0}^{1} d \tau \tau^{N-1} \sigma^{I},
$$

where the $\sigma^{I}$ are now known for both DY and the Higgs boson production up to the $\mathrm{N}^{3} \mathrm{LO}$ level in the threshold limit $[14,15,18]$. In the threshold limit, $N \rightarrow \infty$, we find the following relation exact to all orders in $\epsilon$,

$$
\hat{\phi}_{d}^{I,(i)}(\epsilon)=\frac{\Gamma(1+i \epsilon)}{\Gamma^{2}\left(1+i \frac{\epsilon}{2}\right)} \hat{\phi}^{I,(i)}(\epsilon),
$$

where $\hat{\phi}^{I,(i)}(\epsilon)$ can be found in [16]. Substituting $Z^{I}, \hat{F}^{I}$, and $\Phi_{d}^{I}$ and $\Gamma_{I I}$ in Eq. (5), and using Eq. (4), we obtain $\Delta_{d, I}^{\mathrm{SV}}$ in powers of $a_{s}\left(\mu_{R}^{2}\right)$ as

$$
\begin{aligned}
\Delta_{d, I}^{\mathrm{SV}}(z)= & \sum_{i=0}^{\infty} a_{s}^{i}\left(\mu_{R}^{2}\right) \Delta_{d, I, i}^{\mathrm{SV}}\left(z, \mu_{R}^{2}\right), \quad \text { where } \\
\Delta_{d, I, i}^{\mathrm{SV}}= & \left.\Delta_{d, I, i}^{\mathrm{SV}}\right|_{\delta \delta} \delta\left(\bar{z}_{1}\right) \delta\left(\bar{z}_{2}\right)+\left.\sum_{j=0}^{2 i-1} \Delta_{d, I, i}^{\mathrm{SV}}\right|_{\delta \mathcal{D}_{j}} \delta\left(\bar{z}_{2}\right) \mathcal{D}_{j} \\
& +\left.\sum_{j=0}^{2 i-1} \Delta_{d, I, i}^{\mathrm{SV}}\right|_{\delta \overline{\mathcal{D}}_{j}} \delta\left(\bar{z}_{1}\right) \overline{\mathcal{D}}_{j}+\left.\sum_{j \Theta k} \Delta_{d, I, i}^{\mathrm{SV}}\right|_{\mathcal{D}_{j} \overline{\mathcal{D}}_{k}} \mathcal{D}_{j} \overline{\mathcal{D}}_{k},
\end{aligned}
$$


with $\mathcal{D}_{i}=\left[\frac{\ln ^{i}\left(1-z_{1}\right)}{\left(1-z_{1}\right)}\right]_{+}, \quad \overline{\mathcal{D}}_{i}=\left[\frac{\ln ^{i}\left(1-z_{2}\right)}{\left(1-z_{2}\right)}\right]_{+}$.

The symbol $j \Subset k$ implies $j, k \geq 0$ and $j+k \leq(2 i-2)$. Terms proportional to $\mathcal{D}$ and/or $\overline{\mathcal{D}}$ in Eq. (12) were obtained in [16] and the first term is possible to calculate as the results for the threshold $\mathrm{N}^{3} \mathrm{LO}$ QCD corrections are now available for DY [15] and the Higgs boson [14] productions.

Setting $\mu_{R}^{2}=\mu_{F}^{2}=q^{2}$, in the following, we present this contribution along with the constants $\overline{\mathcal{G}}_{d, i}^{I, k}$ that are needed to determine the soft distribution function $\Phi_{d}^{I}$ up to $\mathrm{N}^{3} \mathrm{LO}$ level using $C_{I}=C_{F}, C_{A}$ for $I=q, g$, respectively.

$$
\begin{aligned}
\overline{\mathcal{G}}_{d, 1}^{I, 1}= & C_{I}\left(-\zeta_{2}\right), \quad \overline{\mathcal{G}}_{d, 1}^{I, 2}=C_{I}\left(\frac{1}{3} \zeta_{3}\right), \quad \overline{\mathcal{G}}_{d, 1}^{I, 3}=C_{I}\left(\frac{1}{80} \zeta_{2}^{2}\right) \\
\overline{\mathcal{G}}_{d, 2}^{I, 1}= & C_{I} C_{A}\left(\frac{2428}{81}-\frac{67}{3} \zeta_{2}-4 \zeta_{2}^{2}-\frac{44}{3} \zeta_{3}\right)+C_{I} n_{f}\left(-\frac{328}{81}+\frac{10}{3} \zeta_{2}+\frac{8}{3} \zeta_{3}\right) \\
\overline{\mathcal{G}}_{d, 2}^{I, 2}= & C_{I} C_{A}\left(-\frac{319}{120} \zeta_{2}^{2}-\frac{71}{3} \zeta_{2} \zeta_{3}+\frac{202}{9} \zeta_{2}+\frac{469}{27} \zeta_{3}+43 \zeta_{5}-\frac{7288}{243}\right)+C_{I} n_{f}\left(\frac{29}{60} \zeta_{2}^{2}-\frac{28}{9} \zeta_{2}-\frac{70}{27} \zeta_{3}+\frac{976}{243}\right) \\
\overline{\mathcal{G}}_{d, 3}^{I, 1}= & C_{I} C_{A}^{2}\left(\frac{17392}{315} \zeta_{2}^{3}+\frac{1538}{45} \zeta_{2}^{2}+\frac{4136}{9} \zeta_{2} \zeta_{3}-\frac{379417}{486} \zeta_{2}+\frac{536}{3} \zeta_{3}^{2}-936 \zeta_{3}-\frac{1430}{3} \zeta_{5}+\frac{7135981}{8748}\right) \\
& +C_{I} C_{A} n_{f}\left(-\frac{1372}{45} \zeta_{2}^{2}-\frac{392}{9} \zeta_{2} \zeta_{3}+\frac{51053}{243} \zeta_{2}+\frac{12356}{81} \zeta_{3}+\frac{148}{3} \zeta_{5}-\frac{716509}{4374}\right)+C_{I} C_{F} n_{f}\left(\frac{152}{15} \zeta_{2}^{2}-40 \zeta_{2} \zeta_{3}\right. \\
& \left.+\frac{275}{6} \zeta_{2}+\frac{1672}{27} \zeta_{3}+\frac{112}{3} \zeta_{5}-\frac{42727}{324}\right)+C_{I} n_{f}^{2}\left(\frac{152}{45} \zeta_{2}^{2}-\frac{316}{27} \zeta_{2}-\frac{320}{81} \zeta_{3}+\frac{11584}{2187}\right) .
\end{aligned}
$$

With $C_{A}=N, C_{F}=\left(N^{2}-1\right) / 2 N, n_{f}=$ no.of flavors and $n_{f, v}$ given in [9], the $\delta \delta$ parts of Eq. (12) for $I=q, g$ are

$$
\begin{aligned}
& \left.\Delta_{d, q, 3}^{\mathrm{SV}}\right|_{\delta \delta}=C_{A}{ }^{2} C_{F}\left(\frac{24352}{315} \zeta_{2}{ }^{3}-\frac{2921}{135} \zeta_{2}{ }^{2}-588 \zeta_{2} \zeta_{3}+\frac{99289}{81} \zeta_{2}-\frac{400}{3} \zeta_{3}{ }^{2}+\frac{125105}{81} \zeta_{3}-204 \zeta_{5}-\frac{1505881}{972}\right) \\
& +C_{A} C_{F}^{2}\left(-\frac{78272}{315} \zeta_{2}^{3}+\frac{137968}{135} \zeta_{2}^{2}+\frac{10736}{9} \zeta_{2} \zeta_{3}-\frac{39865}{27} \zeta_{2}+\frac{1264}{3} \zeta_{3}^{2}-\frac{5972}{3} \zeta_{3}-\frac{7624}{9} \zeta_{5}+\frac{74321}{36}\right) \\
& +C_{A} C_{F} n_{f}\left(-\frac{2828}{135} \zeta_{2}^{2}+\frac{272}{3} \zeta_{2} \zeta_{3}-\frac{12112}{27} \zeta_{2}-\frac{19888}{81} \zeta_{3}-8 \zeta_{5}+\frac{110651}{243}\right) \\
& +C_{F}{ }^{3}\left(\frac{90016}{315} \zeta_{2}{ }^{3}-\frac{3164}{5} \zeta_{2}^{2}-160 \zeta_{2} \zeta_{3}+\frac{1403}{3} \zeta_{2}+\frac{736}{3} \zeta_{3}^{2}-460 \zeta_{3}+1328 \zeta_{5}-\frac{5599}{6}\right) \\
& +C_{F}^{2} n_{f}\left(-\frac{19408}{135} \zeta_{2}^{2}-\frac{1472}{9} \zeta_{2} \zeta_{3}+\frac{5848}{27} \zeta_{2}+360 \zeta_{3}-\frac{224}{9} \zeta_{5}-\frac{421}{3}\right) \\
& +C_{F} n_{f}^{2}\left(\frac{592}{135} \zeta_{2}^{2}+\frac{2816}{81} \zeta_{2}-\frac{304}{81} \zeta_{3}-\frac{7081}{243}\right)+C_{F}\left(\frac{N^{2}-4}{N}\right) n_{f, v}\left(-\frac{4}{5} \zeta_{2}^{2}+20 \zeta_{2}+\frac{28}{3} \zeta_{3}-\frac{160}{3} \zeta_{5}+8\right), \\
& \left.\Delta_{d, g, 3}^{\mathrm{SV}}\right|_{\delta \delta}=C_{A}{ }^{3}\left(\frac{12032}{105} \zeta_{2}{ }^{3}+\frac{40432}{135} \zeta_{2}{ }^{2}-88 \zeta_{2} \zeta_{3}+\frac{41914}{27} \zeta_{2}+\frac{1600}{3} \zeta_{3}{ }^{2}-\frac{54820}{27} \zeta_{3}+\frac{1364}{9} \zeta_{5}+\frac{215131}{81}\right) \\
& +C_{A}^{2} n_{f}\left(\frac{1240}{27} \zeta_{2}^{2}-272 \zeta_{2} \zeta_{3}-\frac{7108}{27} \zeta_{2}+\frac{2536}{27} \zeta_{3}+\frac{1192}{9} \zeta_{5}-\frac{98059}{81}\right) \\
& +C_{A} C_{F} n_{f}\left(\frac{176}{45} \zeta_{2}^{2}+288 \zeta_{2} \zeta_{3}-\frac{2270}{9} \zeta_{2}+400 \zeta_{3}+160 \zeta_{5}-\frac{63991}{81}\right) \\
& +C_{A} n_{f}^{2}\left(-\frac{208}{15} \zeta_{2}^{2}-\frac{64}{3} \zeta_{2}+\frac{112}{3} \zeta_{3}+\frac{2515}{27}\right)+C_{F}^{2} n_{f}\left(\frac{592}{3} \zeta_{3}-320 \zeta_{5}+\frac{608}{9}\right) \\
& +C_{F} n_{f}^{2}\left(-\frac{32}{45} \zeta_{2}^{2}-\frac{184}{9} \zeta_{2}-\frac{224}{3} \zeta_{3}+\frac{8962}{81}\right) \text {. }
\end{aligned}
$$


TABLE I. Relative contributions of pure $\mathrm{N}^{3} \mathrm{LO}$ terms.

\begin{tabular}{ccccccccccccccccc}
\hline \hline & $\delta \delta$ & $\delta \overline{\mathcal{D}}_{0}$ & $\delta \overline{\mathcal{D}}_{1}$ & $\delta \overline{\mathcal{D}}_{2}$ & $\delta \overline{\mathcal{D}}_{3}$ & $\delta \overline{\mathcal{D}}_{4}$ & $\delta \overline{\mathcal{D}}_{5}$ & $\mathcal{D}_{0} \overline{\mathcal{D}}_{0}$ & $\mathcal{D}_{0} \overline{\mathcal{D}}_{1}$ & $\mathcal{D}_{0} \overline{\mathcal{D}}_{2}$ & $\mathcal{D}_{0} \overline{\mathcal{D}}_{3}$ & $\mathcal{D}_{0} \overline{\mathcal{D}}_{4}$ & $\mathcal{D}_{1} \overline{\mathcal{D}}_{1}$ & $\mathcal{D}_{1} \overline{\mathcal{D}}_{2}$ & $\mathcal{D}_{1} \overline{\mathcal{D}}_{3}$ & $\mathcal{D}_{2} \overline{\mathcal{D}}_{2}$ \\
\hline$\%$ & 73.3 & 16.0 & 9.1 & 31.4 & 1.0 & -9.9 & -23.1 & -13.7 & -10.7 & -0.3 & 3.1 & 7.3 & -0.2 & 3.8 & 8.6 & 4.2 \\
\hline \hline
\end{tabular}

We present the relative contributions in percentage of the pure $\mathrm{N}^{3} \mathrm{LO}$ terms in Eq. (12) with respect to $\Delta_{d, g, 3}^{\mathrm{SV}}$, for rapidity $Y=0$ in Table $\mathrm{I}$. The notation $\mathcal{D}_{i} \overline{\mathcal{D}}_{j}$ corresponds to the sum of the contributions coming from $\mathcal{D}_{i} \overline{\mathcal{D}}_{j}$ and $\mathcal{D}_{j} \overline{\mathcal{D}}_{i}$. We have used $\sqrt{s}=14 \mathrm{TeV}$ for the LHC, $G_{F}=$ $4541.68 \mathrm{pb}$, the $Z$ boson mass $m_{Z}=91.1876 \mathrm{GeV}$, top quark mass $m_{t}=173.4 \mathrm{GeV}$ and the Higgs boson mass $m_{H}=125.5 \mathrm{GeV}$ throughout. For the Higgs boson production, we use the effective theory where top quark is integrated out in the large $m_{t}$ limit. The strong coupling constant $\alpha_{s}\left(\mu_{R}^{2}\right)$ is evolved using the 4-loop RG equations with $\alpha_{s}^{\mathrm{N}^{3} \mathrm{LO}}\left(m_{Z}\right)=0.117$ and for parton density sets we use MSTW 2008NNLO [19], as $\mathrm{N}^{3} \mathrm{LO}$ evolution kernels are not yet available. In [20], Forte et al. pointed out that the Higgs boson cross sections will remain unaffected with this shortcoming. However, for the DY process, it is not clear whether the same will be true. We find that the contribution from the $\delta\left(\bar{z}_{1}\right) \delta\left(\bar{z}_{2}\right)$ part is the largest. The dependence on the renormalization and factorization scales can by studied by varying them in the range $\left(m_{H} / 2\right)<\mu_{R}, \mu_{F}<2 m_{H}$. We find that the inclusion of the threshold correction at $\mathrm{N}^{3} \mathrm{LO}$ further reduces their dependence. For the inclusive Higgs boson production, we find that about $50 \%$ of exact NNLO contribution comes from threshold NLO and NNLO terms. It increases to $80 \%$ if we use exact NLO and threshold NNLO terms. Hence, it is expected that the rapidity distribution of the Higgs boson will receive a significant contribution from the threshold region compared to inclusive rate due to the soft emission over the entire range of $Y$. Our numerical study with threshold enhanced NNLO rapidity distribution confirms our expectation. Comparing our threshold NNLO results against exact NNLO distribution using the FEHiP [21] code, we find that about $90 \%$ of exact NNLO distribution comes from the threshold region as can be seen from Table II, in accordance with [22], where it was shown that for low $\tau\left(m_{H}^{2} / s \approx 10^{-5}\right)$ values the threshold terms are dominant, thanks to the inherent property of the matrix element, which receives the largest radiative corrections from the phase-space points corresponding to Born kinematics. Here we have used the exact results up to the NLO level. Because of an inherent ambiguity in the definition of the partonic cross section at threshold one can multiply a factor $z g(z)$, where $z=\tau / x_{1} x_{2}$ and $\lim _{z \rightarrow 1} g(z)=1$, with the partonic flux and divide the same in the partonic cross section for an inclusive rate. In $[23,24]$ this was exploited to take into account the subleading collinear logs also, thereby making the threshold approximation a better one. Recently, Anastasiou et al. used this in [14] to modify the partonic flux keeping the partonic cross section unaltered to improve the threshold effects. Following [14,25], we introduce $G\left(z_{1}, z_{2}\right)$ such that $\lim _{z_{1}, z_{2} \rightarrow 1} G=1$ in (2):

$$
\begin{gathered}
W^{I}=\sum_{a b=q, \bar{q}, g} \int_{x_{1}^{0}} \frac{d z_{1}}{z_{1}} \int_{x_{2}^{0}}^{1} \frac{d z_{2}}{z_{2}} \mathcal{H}_{a b}^{I} G\left(z_{1}, z_{2}\right) \\
\lim _{z_{1}, z_{2} \rightarrow 1}\left[\frac{\Delta_{d, a b}^{I}\left(z_{1}, z_{2}\right)}{G\left(z_{1}, z_{2}\right)}\right] .
\end{gathered}
$$

We also find that with the choice $G\left(z_{1}, z_{2}\right)=z_{1}^{2} z_{2}^{2}$, the threshold NNLO results are remarkably close to the exact ones for the entire range of $Y$ [see Table II, denoted by $(A)$ ]. This clearly demonstrates the dominance of threshold contributions to rapidity distribution of the Higgs boson production at the NNLO level. Assuming that the trend will not change drastically beyond NNLO, we present numerical values for $\mathrm{N}^{3} \mathrm{LO}$ distributions for $G\left(z_{1}, z_{2}\right)=1, z_{1}^{2} z_{2}^{2}$, respectively, as $\mathrm{N}^{3} \mathrm{LO}_{\mathrm{SV}}$ and $\mathrm{N}^{3} \mathrm{LO}_{\mathrm{SV}}(A)$ in Table II. The threshold $\mathrm{N}^{3} \mathrm{LO}$ terms give $6 \%(Y=0)$ to $12 \%(Y=3.6)$ additional correction over the NNLO contribution to the inclusive DY production. Finally, in Table II, we have presented $K 3=\mathrm{N}^{3} \mathrm{LO}_{\mathrm{SV}} / \mathrm{LO}$ as a function of $Y$ in order to demonstrate the sensitivity of higher order effects to the rapidity $Y$.

To summarize, we present full threshold enhanced $\mathrm{N}^{3} \mathrm{LO}$ QCD corrections to rapidity distributions of the dilepton

TABLE II. Contributions of exact NNLO, $\mathrm{NNLO}_{\mathrm{SV}}, \mathrm{N}^{3} \mathrm{LO}_{\mathrm{SV}}$, and $K 3$.

\begin{tabular}{lrrrrrrrrrr}
\hline \hline$Y$ & 0.0 & 0.4 & 0.8 & 1.2 & 1.6 & 2.0 & 2.4 & 2.8 & 3.2 \\
\hline NNLO $_{N N L O}$ & 11.21 & 10.96 & 10.70 & 9.13 & 7.80 & 6.10 & 4.23 & 2.66 & 1.40 & 0.54 \\
$\mathrm{NNLO}_{\mathrm{SV}}(\mathrm{A})$ & 9.81 & 9.61 & 8.99 & 8.00 & 6.71 & 5.21 & 3.66 & 2.25 & 1.14 & 0.42 \\
$\mathrm{~N}^{3} \mathrm{LO}_{\mathrm{SV}}$ & 10.67 & 10.46 & 9.84 & 8.82 & 7.48 & 5.90 & 4.24 & 2.69 & 1.42 & 0.56 \\
$\mathrm{~N}^{3} \mathrm{LO}_{\mathrm{SV}}(\mathrm{A})$ & 11.62 & 11.36 & 11.07 & 9.44 & 8.04 & 6.27 & 4.33 & 2.70 & 1.40 & 0.53 \\
$K 3$ & 11.88 & 11.62 & 11.33 & 9.70 & 8.30 & 6.51 & 4.54 & 2.88 & 1.53 & 0.60 \\
\hline \hline
\end{tabular}


pair in the DY process and of the Higgs boson in gluongluon fusion at the LHC. We show that the infrared structure of QCD amplitudes, in particular, their factorization properties, along with Sudakov resummation of soft gluons and renormalization group invariance provide an elegant framework to compute these threshold corrections systematically for rapidity distributions order by order in QCD perturbation theory. The recent $\mathrm{N}^{3} \mathrm{LO}$ results for inclusive DY and Higgs boson production cross sections at the threshold provide crucial ingredients to obtain $\delta\left(\bar{z}_{1}\right) \delta\left(\bar{z}_{2}\right)$ contribution of their rapidity distributions for the first time. We find that this contribution numerically dominates over the rest of the terms in $\Delta_{d, g, 3}^{\mathrm{SV}}$ at the LHC. Inclusion of $\mathrm{N}^{3} \mathrm{LO}$ contributions reduces the scale dependence further. We also demonstrate the dominance of the threshold contribution to rapidity distributions by comparing it against the exact NNLO for two different choices of $G\left(z_{1}, z_{2}\right)$. Finally, we find that threshold $\mathrm{N}^{3} \mathrm{LO}$ rapidity distribution with $G\left(z_{1}, z_{2}\right)=1, z_{1}^{2} z_{2}^{2}$ shows a moderate effect over NNLO distribution.

We thank F. Petriello for providing the FEHiP code and fruitful discussions. T. A., M. K. M., and N. R. thank IMSc for providing hospitality. We thank M. Mahakhud for discussion. The work of T. A., M. K. M., and N. R. has been partially supported by funding from RECAPP, DAE, Government of India.

"taushif@hri.res.in

†mandal@hri.res.in

*narayan@hri.res.in

§ravindra@imsc.res.in

[1] S. D. Drell and T. M. Yan, Phys. Rev. Lett. 25, 316 (1970); 25, 902 (1970).

[2] A. A. Affolder et al. (CDF Collaboration), Phys. Rev. D 63, 011101 (2000).

[3] F. Abe et al. (CDF Collaboration), Phys. Rev. Lett. 81, 5754 (1998).

[4] G. Aad et al. (ATLAS Collaboration), Phys. Lett. B 716, 1 (2012); S. Chatrchyan et al. (CMS Collaboration), Phys. Lett. B 716, 30 (2012).

[5] J. Kubar-Andre and F. E. Paige, Phys. Rev. D 19, 221 (1979); G. Altarelli, R. K. Ellis, and G. Martinelli, Nucl. Phys. B143, 521 (1978); B146, 544(E) (1978); B. Humpert and W. L. van Neerven, Nucl. Phys. B184, 225 (1981); T. Matsuura and W. L. van Neerven, Z. Phys. C 38, 623 (1988); T. Matsuura, S. C. van der Marck, and W. L. van Neerven, Nucl. Phys. B319, 570 (1989); R. Hamberg, W. L. van Neerven, and T. Matsuura, Nucl. Phys. B359, 343 (1991); B644, 403 (2002).

[6] S. Dawson, Nucl. Phys. B359, 283 (1991); A. Djouadi, M. Spira, and P. M. Zerwas, Phys. Lett. B 264, 440 (1991);
M. Spira, A. Djouadi, D. Graudenz, and P. M. Zerwas, Nucl. Phys. B453, 17 (1995); R. V. Harlander and W. B. Kilgore, Phys. Rev. D 64, 013015 (2001); S. Catani, D. de Florian, and M. Grazzini, J. High Energy Phys. 05 (2001) 025; 07 (2003) 028; R. V. Harlander and W. B. Kilgore, Phys. Rev. Lett. 88, 201801 (2002); C. Anastasiou and K. Melnikov, Nucl. Phys. B646, 220 (2002); V. Ravindran, J. Smith, and W. L. van Neerven, Nucl. Phys. B665, 325 (2003).

[7] C. Anastasiou, L. J. Dixon, K. Melnikov, and F. Petriello, Phys. Rev. Lett. 91, 182002 (2003); C. Anastasiou, K. Melnikov, and F. Petriello, Phys. Rev. Lett. 93, 262002 (2004).

[8] S. Moch, J. A. M. Vermaseren, and A. Vogt, J. High Energy Phys. 08 (2005) 049; Phys. Lett. B 625, 245 (2005).

[9] P. A. Baikov, K. G. Chetyrkin, A. V. Smirnov, V. A. Smirnov, and M. Steinhauser, Phys. Rev. Lett. 102, 212002 (2009).

[10] T. Gehrmann, E. W. N. Glover, T. Huber, N. Ikizlerli, and C. Studerus, J. High Energy Phys. 06 (2010) 094; 11 (2010) 102.

[11] S. Moch, J. A. M. Vermaseren, and A. Vogt, Nucl. Phys. B688, 101 (2004); B691, 129 (2004).

[12] T. Inami, T. Kubota, and Y. Okada, Z. Phys. C 18, 69 (1983); K. G. Chetyrkin, B. A. Kniehl, and M. Steinhauser, Phys. Rev. Lett. 79, 353 (1997); Nucl. Phys. B510, 61 (1998).

[13] S. Moch and A. Vogt, Phys. Lett. B 631, 48 (2005); E. Laenen and L. Magnea, Phys. Lett. B 632, 270 (2006); A. Idilbi, X.-d. Ji, J.-P. Ma, and F. Yuan, Phys. Rev. D 73, 077501 (2006); V. Ravindran, Nucl. Phys. B746, 58 (2006); B752, 173 (2006).

[14] C. Anastasiou, C. Duhr, F. Dulat, E. Furlan, T. Gehrmann, F. Herzog, and B. Mistlberger, Phys. Lett. B 737, 325 (2014).

[15] T. Ahmed, M. Mahakhud, N. Rana, and V. Ravindran, Phys. Rev. Lett. 113, 112002 (2014).

[16] V. Ravindran, J. Smith, and W. L. van Neerven, Nucl. Phys. B767, 100 (2007); V. Ravindran and J. Smith, Phys. Rev. D 76, 114004 (2007).

[17] V. Ravindran, Nucl. Phys. B752, 173 (2006).

[18] Y. Li, A. von Manteuffel, R. M. Schabinger, and H. X. Zhu, Phys. Rev. D 90, 053006 (2014).

[19] A. D. Martin, W. J. Stirling, R. S. Thorne, and G. Watt, Eur. Phys. J. C 63, 189 (2009).

[20] S. Forte, A. Isgr, and G. Vita, Phys. Lett. B 731, 136 (2014).

[21] C. Anastasiou, K. Melnikov, and F. Petriello, Nucl. Phys. B724, 197 (2005).

[22] T. Becher, M. Neubert, and G. Xu, J. High Energy Phys. 07 (2008), 030.

[23] S. Catani, D. de Florian, M. Grazzini, and P. Nason, J. High Energy Phys. 07 (2003) 028.

[24] M. Kramer, E. Laenen, and M. Spira, Nucl. Phys. B511, 523 (1998).

[25] F. Herzog and B. Mistlberger, arXiv:1405.5685. 